\title{
Tracking the US business cycle with a singular spectrum analysis
}

\author{
Miguel de Carvalho ${ }^{\mathrm{a}, *}$, Paulo C. Rodrigues ${ }^{\mathrm{b}}$, António Rua ${ }^{\mathrm{c}}$ \\ ${ }^{a}$ Ecole Polytechnique Fédérale de Lausanne, Switzerland \\ ${ }^{\mathrm{b}}$ Universidade Nova de Lisboa, Portugal \\ ${ }^{\mathrm{c}}$ Banco de Portugal, Portugal
}

\section{A R T I C L E I N F O}

\section{Article history:}

Received 17 January 2011

Received in revised form

30 August 2011

Accepted 2 September 2011

Available online 14 September 2011

\section{JEL classification:}

C50

E32

Keywords:

Band-pass filter

Principal components

Singular spectrum analysis

US business cycle

\begin{abstract}
A B S T R A C T
In this paper we resort to singular spectrum analysis to disentangle the US GDP into several underlying components of interest. The business cycle indicator yielded through this method is shown to bear a resemblance with band-pass filtered output, and our results suggest it possesses better revision performance than other commonly applied filters.
\end{abstract}

(C) 2011 Elsevier B.V. All rights reserved.

\section{Introduction}

Among the most employed methods to extract the latent cyclical component of an economic series lies the method proposed by Hodrick and Prescott (1997). Despite its popularity this is a high-pass filter and thus incorporates excessive noisy movements in the cyclical component. Baxter and King (1999) recommend instead the use of a band-pass filter which suppresses a spectrum of frequencies, and although their method is unable to produce end-of-sample estimates, which are particularly precious for policymaking, Christiano and Fitzgerald (2003) proposed a related method which solves this. Although these methods are among the most employed, the literature includes many possibilities. For example, Yogo (2008) uses multiresolution wavelet analysis to track cycles, Harvey and Trimbur (2003) suggest a class of filters based on unobserved components time series models, and Azevedo et al. (2006) propose a multivariate filter which uses information from many sources.

Here we employ singular spectrum analysis to disentangle the US GDP into several components of interest. Singular spectrum analysis is an extension of principal components for time series,

\footnotetext{
* Correspondence to: Ecole Polytechnique Fédérale de Lausanne, MATHAA-FSBEPFL, CH-1015 Lausanne, Switzerland. Fax: +41 216934250.

E-mail address: Miguel.Carvalho@epfl.ch (M. de Carvalho).
}

with applications in climatology (Allen and Smith, 1996), geophysics (Kondrashov and Ghil, 2006), and meteorology (Paegle et al., 2000); other applications include forecasting (Hassani et al., 2009). The business cycle indicator yielded through this method resembles band-pass filtered output, it is in line with the contraction periods dated by the NBER, and a real-time exercise indicates it possesses better revision performance than some competing filters.

\section{Singular spectrum analysis in a nutshell}

\subsection{Modus operandi of singular spectrum analysis}

The method includes the phases of decomposition and reconstruction (Golyandina et al., 2001). Decomposition subdivides into the steps of embedding and singular value decomposition.

Embedding.

The main concept in this step is the trajectory matrix, i.e., a lagged version of the original series $\mathbf{y}=\left[\begin{array}{lll}y_{1} & \cdots & y_{n}\end{array}\right]^{\prime}$, which is defined as

$\mathbf{Y}=\left[\begin{array}{cccc}y_{1} & y_{2} & \cdots & y_{\kappa} \\ y_{2} & y_{3} & \cdots & y_{\kappa+1} \\ \vdots & \vdots & \ddots & \vdots \\ y_{l} & y_{l+1} & \cdots & y_{l+(\kappa-1)}\end{array}\right]$, 

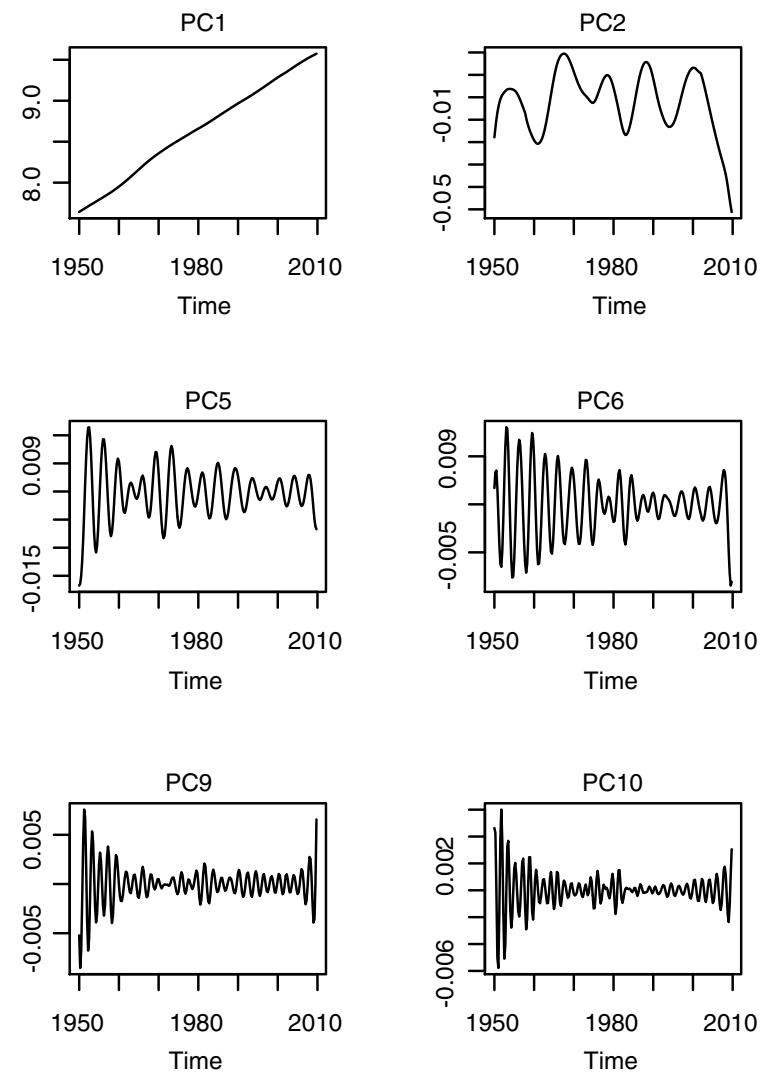
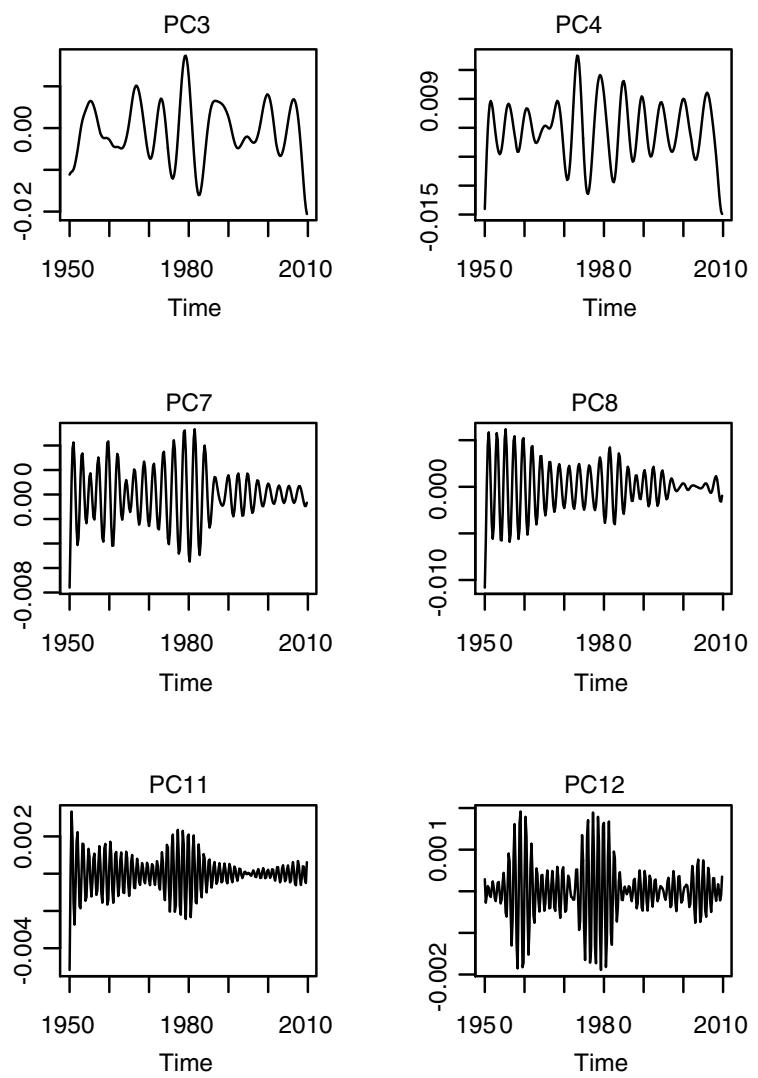

Fig. 1. The first 12 principal components.

with $\kappa=n-l+1$. We refer to each vector $\mathbf{y}_{i}=\left[\begin{array}{lll}y_{i} & \cdots & y_{l+(i-1)}\end{array}\right]^{\prime}$, as a window; the window length $l$, is a parameter defined by the user.

Singular value decomposition

From an eigenanalysis of $\mathbf{Y Y}^{\prime}$ we collect the eigenvalues $\lambda_{1} \geq$ $\cdots \geq \lambda_{d}$, where $d=\operatorname{rank}\left(\mathbf{Y} \mathbf{Y}^{\prime}\right)$, and the corresponding left and right singular vectors, respectively denoted by $\mathbf{w}_{i}$ and $\mathbf{v}_{i}$. Through the decomposition made in this step we can approximate Y through

$\mathbf{Y} \approx \sum_{i=1}^{d} \sqrt{\lambda_{i}} \mathbf{w}_{i} \mathbf{v}_{i}^{\prime}$

We now turn to the second phase of the method, reconstruction; this includes grouping and diagonal averaging.

Grouping.

In this step we select the first $m$ leading eigentriples associated to the signal and exclude the remaining $(d-m)$ associated to the noise. Thus, we perform a proper selection of $m$, to disentangle $\mathbf{Y}$ into

$\mathbf{Y}=\sum_{i=1}^{m} \sqrt{\lambda_{i}} \mathbf{w}_{i} \mathbf{v}_{i}^{\prime}+\boldsymbol{\varepsilon}$,

where $\boldsymbol{\varepsilon}$ denotes an error term, and the remainder summands represents the signal. This is typically done through readjusted methods for selecting the number of principal components.

Diagonal averaging.

The central idea in this step is the reconstruction of the deterministic component of the series. Here we reverse the process done so far, returning to a reconstructed variant of the trajectory matrix, and thus the deterministic component of the series. An optimal way to do this is to average over all the elements of the several 'antidiagonals'. Formally, consider the linear space $\mathcal{M}_{l, \kappa}$ formed by the collection of all the $l \times \kappa$ matrices, let $\left\{\mathbf{h}_{l}\right\}_{l=1}^{n}$ denote the canonical basis of $\mathbb{R}^{n}$, and consider the matrix $\mathbf{X}=\left[x_{i, j}\right] \in$ $\mathcal{M}_{l \times \kappa}$. The diagonal averaging procedure is hence carried on by the mapping $\overline{\mathbb{D}}: \mathcal{M}_{l \times \kappa} \rightarrow \mathbb{R}^{n}$ defined as

$\overline{\mathbb{D}}(\mathbf{X})=\sum_{w=2}^{\kappa+l} \mathbf{h}_{w-1} \sum_{(i, j) \in \mathcal{A}_{w}} \frac{x_{i, j}}{\left|\mathcal{A}_{w}\right|}$.

Here $|\cdot|$ denotes the cardinal operator, and $\mathcal{A}_{w}=\{(i, j): i+j=w\}$. We can now write the deterministic component of $\mathbf{y}$ as

$\widetilde{\mathbf{y}}=\overline{\mathbb{D}}\left(\sum_{i \in I} \sqrt{\lambda_{i}} \mathbf{w}_{i} \mathbf{v}_{i}^{\prime}\right)$.

\section{Measuring the US business cycle}

\subsection{Tracking the business cycle}

Data from the US GDP were gathered from Thompson Financial Datastream; the time horizon ranges from 1950 (Q1) to 2009 (Q4). As it is standard in related literature, the business cycle is considered as the cyclical component whose recurring movements range from 6 to 32 quarters. This conception is handy for window length selection and for electing the principal components to be discarded. Since we are interested in the follow-up of regular dynamics of up to 8 years, setting a window length of 32 quarters becomes the natural choice. This leads to the components depicted in Fig. 1. The above-mentioned business cycle definition enables us to dispense with the first two components: PC1 is linked to a 


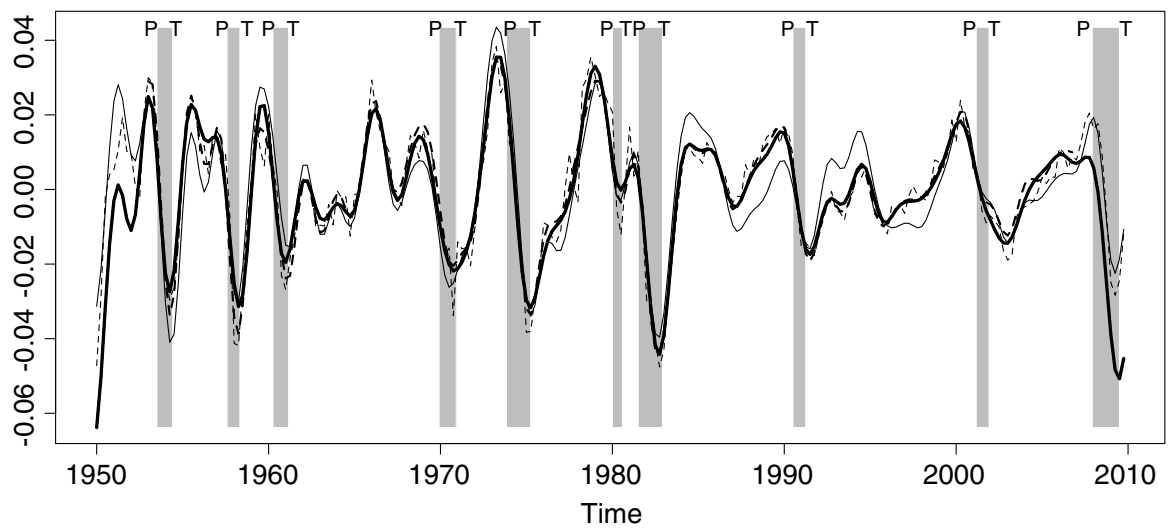

Fig. 2. Comparison of Indicators: CRR (-), CF (-), HP (- - -), and BK (- -).
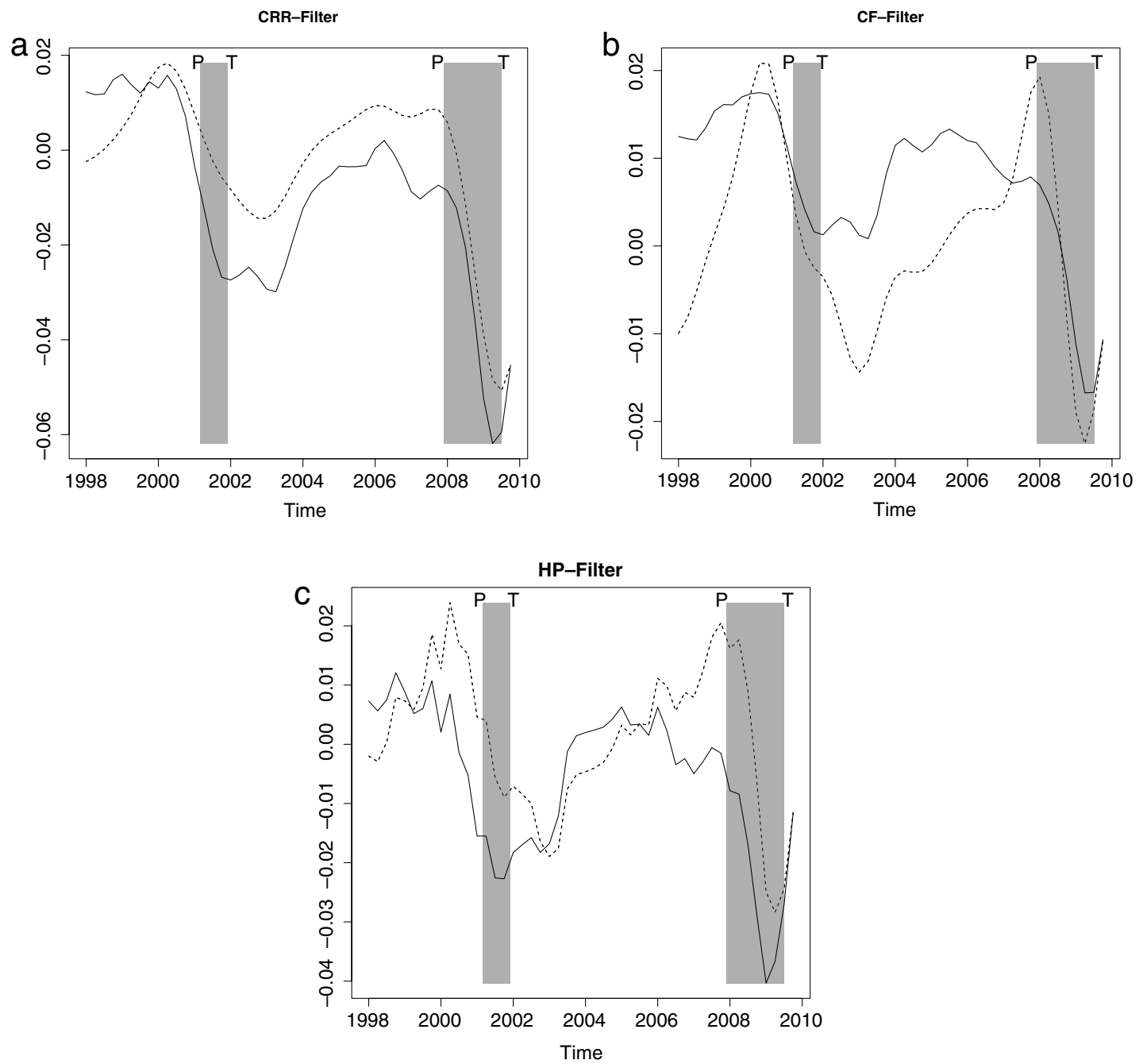

Fig. 3. Comparison of final (-) and real-time estimates (- - -).

slow-moving component (trend); PC2 is associated to movements with a frequency noticeably larger than 32 quarters. Components above the ninth are not considered relevant, as they take control of short fluctuations markedly below 6 quarters. Our indicator, henceforth CRR-filter, is thus composed by summing the remainder (3-9) components.
Fig. 2 represents the business cycle obtained with the CRRfilter against the indicators yield by the methods of: Christiano-Fitzgerald (CF); Baxter-King (BK); and Hodrick-Prescott (HP). Some comments are in order. First, the aforementioned highpass features of the HP-filter are visible in its bumpy behavior. Second, both the CF and BK have a similar behavior given their 
Table 1

Reliability report.

\begin{tabular}{lll}
\hline & Reliability statistic & Noise-to-signal \\
\cline { 2 - 3 } & Correlation & 0.844 \\
\hline Hodrick-Prescott & 0.649 & 0.750 \\
Christiano-Fitzgerald & 0.669 & 0.583 \\
Carvalho-Rodrigues-Rua & 0.893 & 0.553 \\
\hline
\end{tabular}

band-pass attributes, but BK-filter is unable to yield end-of-sample estimates. Lastly, our indicator possesses a similar behavior to the remainder and hence it can be thought of as an alternative method for characterizing the cyclical dynamics of economic activity, and it is in line with the contractions and expansions dated by the NBER. Our indicator yields lower estimates than comparing methods at both ends of the sample. This agrees with Stock and Watson (1999, Figure 2.5), who also classified our first contraction period as the one with lowest values in the last 50 years of the last millennium; the severity of the latest contraction period also suggests that the remainder filters may be excessively optimistic here.

\subsection{A real-time exercise}

From the policymaker's stance a feature of remarkable importance is the reliability of real-time estimates of the business cycle indicator (Orphanides and van Norden, 2002). By a real-time estimate we mean the business cycle estimate, conditional on the information set available at such point in time. Ex post revision of the estimates is typically due to either published data revision, or to recomputations given the arrival of further data on subsequent quarters. Here, a fixed data set is used so that the unique source of revision is the latter. As advocated by Orphanides and van Norden (2002), recomputations are responsible for a large share of recurrent revision in the estimates. The period under consideration is (Q1)1998-(Q4)2009 and corresponds to 20\% of the sample size. In Fig. 3 we plot real-time business cycle estimates against final estimates.

We report in Table 1 a set of revision realiability measures. The real-time performance of the HP-filter is in the overall dominated by the CF-filter, and our indicator dominates in all the abovementioned measures the CF and HP filters. The correlation of the CRR-filter is almost 0.9 and hence much higher than the ones obtained by the HP and CF filters ( 0.65 and 0.67 , respectively). The noise-to-signal ratio is quite lower reinforcing the relative information content of the real-time estimates obtained with the CRR-filter. The share of time that the real-time estimates of the CRR-filter have the same sign as final estimates are higher in comparison with the HP and CF filters. Further results, available from the authors, suggest better revision of our indicator in comparison with wavelet-based filter by Yogo (2008) and the unobserved components model by Harvey and Trimbur (2003).

\section{Conclusion}

This paper introduces the CRR-filter, a business cycle indicator based on singular spectrum analysis which is shown to bear a resemblance with band-pass filtered output. Since the end-ofsample behavior is frequently a thorny issue in business cycle assessment, a real-time estimation exercise is here executed to compare the reliability of the HP, CF, and CRR filters. Our results suggest that the indicator proposed herein is endowed with a better revision performance than other filters often applied in the literature.

\section{Acknowledgments}

M. de C. acknowledges financial support from Banco de Portugal and Centro de Matemática e Aplicações, Universidade Nova de Lisboa.

\section{References}

Allen, M., Smith, L., 1996. Monte Carlo SSA: detecting irregular oscillations in the presence of colored noise. Journal of Climate 9, 3373-3404.

Azevedo, J., Koopman, S. Rua, A., 2006. Tracking the business cycle of the Euro area: a multivariate model-based bandpass filter. Journal of Business \& Economic Statistics 24, 278-290.

Baxter, M., King, R., 1999. Measuring business cycles: approximate band-pass filters for economic time series. Review of Economics and Statistics 81, 575-593.

Christiano, L., Fitzgerald, T., 2003. The band-pass filter. International Economic Review 44, 435-465.

Golyandina, N., Nekrutkin, V., Zhigljavsky, A., 2001. Analysis of Time Series Structure: SSA and Related Techniques. Chapman \& Hall, CRC, London.

Harvey, A. Trimbur, T. 2003. General model-based filters for extracting cycles and trends in economic time series. Review of Economics and Statistics 85, 244-255.

Hassani, H., Heravi, S., Zhigljavsky, A., 2009. Forecasting European industrial production with singular spectrum analysis. International Journal of Forecasting $25,103-118$.

Hodrick, R.J., Prescott, E., 1997. Postwar US business cycles: an empirical investigation. Journal of Money, Credit and Banking 29, 1-16.

Kondrashov, D., Ghil, M., 2006. Spatio-temporal filling of missing points in geophysical data sets. Nonlinear Processes in Geophysics 13, 151-159.

Orphanides, A., van Norden, S., 2002. The unreliability of output-gap estimates in real time. Review of Economics and Statistics 84, 569-583.

Paegle, J., Byerle, L., Mo, K., 2000. Intraseasonal modulation of South American summer precipitation. Monthly Weather Review 128, 837-850.

Stock, J., Watson, M., 1999. Business cycle fluctuations in US macroeconomic time series. In: Taylor, J., Woodford, M. (Eds.), Handbook of Macroeconomics, vol. 1. pp. 3-64.

Yogo, M., 2008. Measuring business cycles: a wavelet analysis of economic time series. Economics Letters 100, 208-212. 